\title{
APROXIMACIÓN AL CONOCIMIENTO PRÁCTICO PERSONAL DE LOS PROFESORES DE MATEMÁTICAS DE EGB
}

\author{
BLANCO NIETO, L. \\ Departamento de Didáctica de las Ciencias Experimentales y de las Matemáticas. \\ Universidad de Extremadura. Badajoz.
}

\section{SUMMARY}

This work is placed in the teacher thinking paradigm and wants to show some outcomes from a research in teacher training in order to study the teachers' personal practical knowledge. We have used the qualitative method, mainly, the classroom observation, the interview, and the stimulated recall. It is specifically about two examples of mathematics teaching: the teaching of mathematical concepts and problem solving in primary schools.

En más de una ocasión hemos justificado alguna actividad haciendo uso del refranero: «Cada maestrillo tiene su librillow. Este dicho popular, de sobra conocido, pone de manifiesto el convencimiento general que tenemos acerca del aprendizaje que nos depara nuestra experiencia. Idea que podemos considerar, así mismo, para los profesionales de la enseñanza.

Sin embargo, es sólo recientemente cuando se acepta que el profesor es un sujeto reflexivo y racional, que toma decisiones en un entorno complejo e incierto, cuyo pensamiento guía y orienta su conducta en clase (Shavelson y Stern 1983, Marcelo 1987). Esta perspectiva está siendo cada vez más considerada en las investigaciones educativas, frente a esa otra, más generalizađa, que considera a los profesionales de la enseñanza como meros técnicos que aplican unos determinados métodos asimilados en su período de formación o durante su práctica docente.

Los profesores en ejercicio van elaborando, como consecuencia de su actividad, un cuerpo de conocimiento, explícito o implícito, que surge de su propia experiencia, de la reflexión y análisis que de ella realizan, así conto de la observación del entorno en el que desarrollan su actividad profesional.
La necesidad de comprender el conocimiento de contenido pedagógico de los profesores de Matemáticas (Livingston y Borko 1989 , p. 37) constituye uno de los objetivos de una investigación desarrollada en la Universidad de Extremadura con profesores de EGB (Blanco 199 la). En ella, considerando técnicas propias de Ia metodología cualitativa, observaciones de clase, grabaciones en audio y vídeo, y entrevistas a los participantes, se analizan estudios de casos en los que se pone de manifiesto el conocimiento práctico que los profesores van adquiriendo como consecuencia de su experiencia docente.

La lectura de las transcripciones correspondientes a las clases y entrevistas, así como el visionado de los vídeos nos permitió delimitar, de acuerdocon nuestros informantes, dos grandes apartados de su actividad docente que constituyen la base del trabajo: Ia enseñanza de los conceptos matemáticos y la resolución de problemas. (Blanco 1991a, p. 121).

Tomando como referencia estos apartados, damos a conocer dos ejemplos concretos del conocimiento práctico personal de uno de nuestros informantes que aparecen claramente reflejados en la fase de «análisis especulativo» (Woods 1987) propia del proceso de investigación seguido (Blanco 1991 a, p. 122). En ambos casos, hemos establecido 
una comparación entre tres fuentes de información: a) transcripciones de clase y grabaciones de vídeos; b) transcripciones de las entrevistas de estimulación del recuerdo (Caiderhead 1981), y c) propuestas sobre Didáctica de las Matemáticas referidas a los apartados anteriores.

Nuestra informante, maestra de segunda etapa de EGB especialista en Matemáticas, ha desarroliado su actividad docente en ambientes, fundamentalmente, rurales que le han arrastrado a un proceso de autoformación y sencille $z$ en sus planteamientos. Su trayectoria profesional nos permite asegurar que su conocimiento pedagógico surge, fundamentalmente, de su actividad docente y de la reflexión que, de ella, haya podido realizar.

«Estudié con el Plan de estudio que se hacía con $4^{\circ}$ y reválida. Las oposiciones las hice en Badajoz en el año 69-70, y a partir de este año, estoy en activo. Los tres primeros cursos estuve en la educación de adultos, en alfabetización, en Campanario, en Castuera. A partir del 4" año me incorporé a la EGB en Alburquerque hasta el año pasado que llegué a Badajoz. A partir de entonces he estado en segunda etapa, menos este año que por cuestiones de necesidad del centro, estoy en $5^{\circ}$.

He estado dando Matemáticas y Ciencias, pero también por necesidad, alguna vez, Lengua, Inglés o «las Marías». Lo que más me gusta son las Matemáticas, además creo que se me da mejor el área de ciencias, prefiero el razonamiento a la memorización.»

\section{EJEMPLO 1.PRINCIPIO DE VARIABILIDAD MATEMATICA}

Hacemos referencia al principio de variabilidad enunciado por Z. P. Dienes cuyo contenido parece ser desarrollado por uno de nuestros informantes aun cuando manifiesta expresamente su desconocimiento sobre el mismo.

En todo el proceso de enseñanza de las Matemáticas Z. P. Dienes (1970) considera necesario tener en cuenta cuatro principios que ayudarían a los alumnos en la comprensión de los conceptos matemáticos: principio dinámico, principio de constructividad, principio de variabilidad matemática y principio de concretización mútíiple.

En el tercero de ellos, expresa: «los conceptos que encierran más de una variable deben ser estudiados mediante experiencias que supongan el manejo del mayor número posible de aquelias variables» (Dienes 1970, p. 31). Es necesario ponerlas de manifiesto expresamente y de forma natural, facilitando al alumno la comprension y abstracción del concepto.

De nuestras observaciones destacamos que los profesores con experiencia hacen referencia, de forma implícita, a distintas variables que aparecen en los conceptos matemáticos que pretenden introducir, escalonándolas de tal manera que el alumno va gradualmente comprendiendo o repasando todos los aspectos que puedan considerarse dentro del concepto estudiado.

Así, observamos que en una de las clases grabadas en las que trabaja sobre operaciones con los números decimales, nuestra informante va haciendo referencia, gradualmente, al significado de los números decimales, de las operaciones, a la colocación de los números con referencia a las unidades semejantes, etc. En una misma sesion, al referirse a la suma de números decimales plantea, entre otras, las siguientes cuestiones:

«Juan Carlos nos va a poner un ejemplo con números decimales sencillitos, y nos va a explicar cómo se suma y por qué.»

« ¿Por qué has colocado estas unidades así, y por ejemplo no lo has hecho así...?»

"¿Por qué las décimas tienen que estar con las décimas?»

"Quiero que cada cual invente la letra de un problema con esas cantidades y con esa operación.»

«Si no hay..., ¿qué se pone cuando no hay? No, no hay centésimas aquí, ¿Qué se pone? ¿Cómo se representa? Vicente, ¿ cómo se representa en Matemáticas cuando no hay algo?»

«Tenías tú 53,6. Alejandro, fíjate, tenía 53,6 y resulta que ha puesto 53,600 , en lugar de 6 pone $600 \ldots$, ipor qué?»

En las entrevistas de estimulación del recuerdo realizadas a partir de las grabaciones de vídeo, hablamos de esta situación y de la relación que encontrabámos con el principio de variabilidad matemática de Dienes (1970). En todo momento, nuestra informante, que considera su práctica docente como la fuente fundamental de su conocimiento, hará referencia a su experiencia y al conocimiento que ticne de los alumnos para decidir sobre las ideas o tipo de actividades a desarrollar. A este respecto, conviene señalar que encontramos una proyección de los comentarios de nuestra experta en las subcategorías de conocimiento de contenido pedagógico en Matemática elemental identificadas en Marks (1989).

«Esto se va haciendo teniendo en cuenta Ios fallos que yo me encuentro al corregir controles, al corregir ejercicios, entonces voy remachando sobre esos fallos.»

«[...] Se aprovecha cualquier circunstancia para recordar cómo se hace...»

«[...] Recupero objetivos que se habían quedado un poco atrasados. La base es corregir errores que se cometen por parte de los alumnos. Esto lo da el conocimiento de ellos.»

«[...] La experiencia te va dando pautas para pararte más en algunos sitios y pasarte otras cosas que no las consideras importantes.» 
En la figura 1 esquematizamos los resultados poniendo de manifiesto la triangulación establecida en el análisis de los datos.

Figura 1

Conocimiento práctico relacionado con el principio de variabilidad de Dienes (1970).

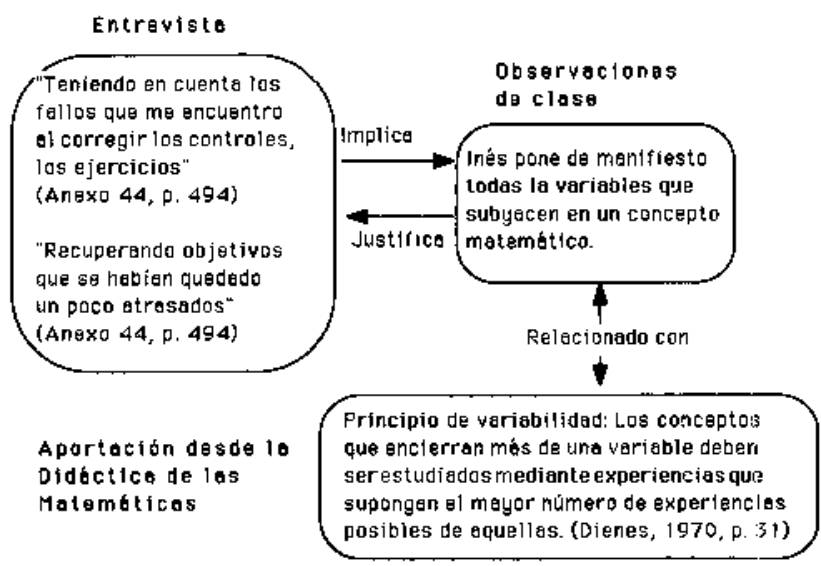

\section{EJEMPLO 2. RESOLUCIÓNDEPROBLEMAS}

Frecuentemente, los profesionales de la enseñanza de las Matemáticas manifestamos nuestra preocupación porque los alumnos aprendan a resolver problemas. A este respecto, se identifica una perspectiva en la enseñanza de la resolución de problemas (Teaching about problem solving) cuyo objetivo principal es que los alumnos lleguen a aprender estrategias entre las que elegir, que deberían ayudarles a idear y llevar a cabo diferentes planes de resolución de problemas (Gaulin 1986 y Schrocder y Lester 1989).

Los profesores que enseñan sobre la resolución de problemas destacan el modelo de Polya $(1949,1985)$ o alguna variación sobre el mismo. Este modelo describe un conjunto de cuatro fases independientes en el proceso de resolución de problemas matemáticos: comprender el problema, concebir un plan, ejecutar el plan y visión retrospectiva. De acuerdo con Polya, los resolutores expertos las usan cuando resuelven problemas matemáticos. La enseñanza sobre la resolución de problemss implica discusión explícita, de los estudiantes, sobre cómo los problemas están siendo resueltos, a fin de que tomen conciencia de su propia progresión (Schroeder y Lester 1989).

En las clases de nuestra informante aparecen momentos perfectamente delimitados en los que manifiesta a los alumnos su objetivo de realizar problemas. Éstos entra rían dentro del apartado de problemas de traducción simple o compleja (Charles y Lester 1982), es decir problemas típicos cuya resolución supone una traducción del texto a una expresión matemática.

En el desarrollo de los mismos manifiesta, explícitamente, dos objetivos. Por una parte, el conocimiento de aspectos de contenidos matemáticos propios del tema que se esté desarrollando; por otra, quiere que sus alumnos aprendan un esquema concreto para la resolución, en general, de los problemas.

La situación estudiada aparece como continuación de la sesión considerada en el ejemplo anterior. En cl comienzo de su realización, comenta diferentes variables conceptuales, o de algoritmo, que aparecerán en los problemas y que ayudarán a los alumnos en la realización y comprensión de los mismos. Así, en la intervención de nuestra informante, referente a los números decimales, que hemos transcrito en el ejemplo 1, obscrvamos que propone a los alumnos que enuncien problemas que se correspondan con una determinada operación, apareciendo esta actividad antes que la propuesta de realizar problemas en los que intervienen las operaciones de números decimales.

Los alumnos habían realizado una actividad que llama ejercicios (práctica de los algoritmos), en la que realizaban operaciones a partir de números dados. En ese momento, diferenciando los dos tipos de actividades, ejercicios y problemas, nuestra profesora se plantea expresamente la resolución de problemas:

«Venga, coged el cuaderno que vamos a hacer ahora un problemita sencillo. Vamos a ver, son problemitas muy sencillos. A ver, sal al encerado. Poned la fecha... Venga, poned ahí: De un depósito [...]»

Cuando aparecen estas orientaciones toma como referencia un esquema de elaboración propia, que expresamente manifiesta en las entrevistas, y que considera adecuado para esta actividad. Tanto en cl desarrollo de las clases como en las entrevistas mantenidas podemos apreciar que el proceso seguido por nuestra informante presenta ciertas similitudes, en diferentes aspectos concretos, con el modelo propuesto por Polya, y que queremos resaltar. A este respecto podemos establecer un cierto paralelismo entre los pasos seguidos por nuestra informante, con diferente denominación, y los referidos anteriormente para Polya.

\section{a) Entender el problema}

Mientras dicta el problema hace algunas preguntas a los alumnos relativas a los conceptos o términos que van surgiendo en el enunciado y que ayudan a la comprensión del mismo.

Una vez terminada la fase de cnunciar cl problema vemos como se dirige a los alumnos y les indica:

«Cuando tenemos escrito un problema, antes de hacer absolutamente nada, ¿qué hay que hacer? [...] Leerlo. Lo primero que vamos a hacer, todo el mundo, es volver a leer el problema... Luis te estoy viendo el lápiz. [...] Leemos el problema para entenderlo.»

El primer objetivo, en esta actividad, se encamina pues a la comprensión deI problema. 


\section{b) Identificación de datos e incógnita}

A continuación marca el segunđo paso en la resolución del problema que debe llevar a los alumnos a la identificación de los datos y las incógnitas, como paso previo para decidir el camino de solución.

«Una vez que lo hemos leído... Vicente, ¿ya has leído el problema? [...] ¿Qué hacemos? ¿Qué pide? ¿Sabemos qué pide el problema? [...] ¿Cuál es la cantidad de agua que hay en el depósito? [...] Eso es lo que nos pide, eso tenemos que tenerlo clarito, porque si no sabemos lo que nos pide el problema, nunca sabremos qué es lo que tendremos que hacer."

\section{c) Señalar un camino y realización del problema}

Es en este momento cuando los alumnos están dispuestos a realizar el problema. Hasta este momento los alumnos habían estado sin escribir nada, sin coger el lápiz, únicamente mirando el enunciado y contestando a las diversas cuestiones que la profesora había ido planteando.

«Eso es lo que nos pide, ...entonces, una vez que ya sabemos qué tenemos que hacer, que hay que averiguar la cantidad de agua que hay en el depósito, vamos a hacer el problema.»

\section{d) Análisis del problema}

Por último, la profesora señala la necesidad de reflexión sobre la solucion del problema. En este caso el alumno se ha equivocado en la resolución del problema y le hace reflexionar sobre su error, intentando que sea el propio alumno el que rectifique:

«Inés, [...] ahora te voy a pedir que vuelvas a leer el problema y que me digas si encuentras algo raro. Pon atención. [...] Antes de hacer nada dime lo raro que tú ves.

Alumno. «Que quedan 128 litros en el depósito.»

Inés. «Quedan 128 litros en el depósito y a ti te sale que en total son...»

Alumno. «59,55».

Inés. ¿Es eso posible? Es imposible que si quedan 128, el total fuera 59,55 . ¿Lo veis? [...] Es imposible. Entonces vamos a averiguar, antes de borrar, ...no borres nada. Vamos a ver dónde has fallado. Otra vez lo lees, despacito y ves dónde fallas.»

A partir de esta reflexión, y del repaso que suele dar al problema una vez concluido, se volverá a enlazar con los aspectos de la teoría matemática (números decimales y operaciones aritméticas elementales) que han ido apareciendo en el problema, y a partir de aquí sacará diferentes conclusiones para cada caso.

Por otra parte, en esta última fase de la resolución del problema pregunta a los alumnos sobre otras formas de hacerlos.
«Vamos a ver. Otro que lo haya hecho de otra manera. Venga, Julián... Dime de qué forma to has hecho.»

En la figura 2 presentamos un esquema que representa el proceso seguido en la resolución de problemas a partir de las observaciones de clase, que como vemos en el apartado $e$ ) coincide con sus manifestaciones en las entrevistas.

Figura 2

Proceso seguido para la resolución de problemas.

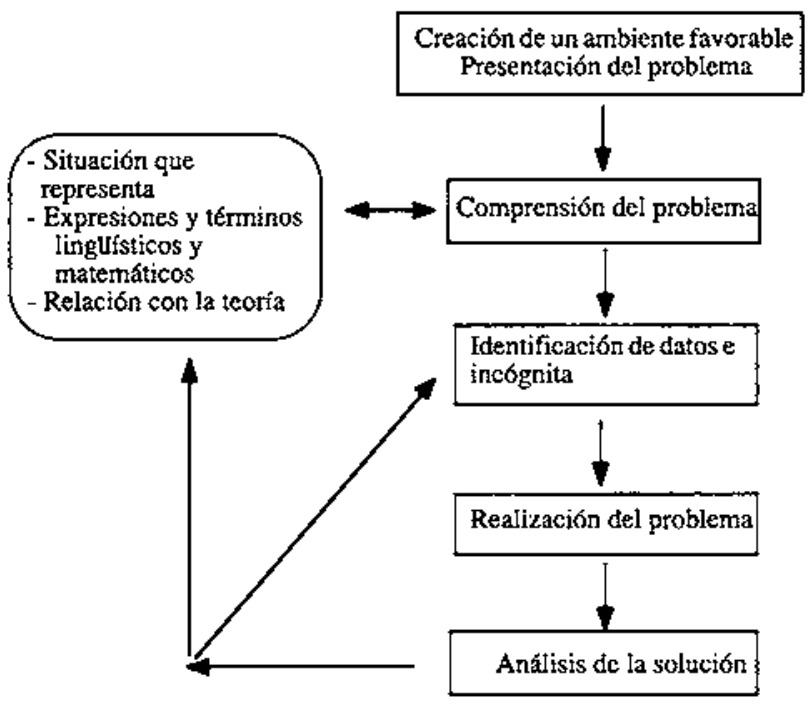

e) Su conocimiento de los pasos anteriores

Como hemos visto, las observaciones de clase nos permiten señalar que basa su metodología para enseñar a resolver problemas en cuatro aspectos, que identifica como: entender el enunciado, identificación de los datos e incógnita, señalar un camino y análisis del problema. Estos aspectos habían sido marcados por esta profesora en la primera entrevista que mantuvimos donde nos expresaba su conocimiento:

«Los niños no saben resolver problemas. Al principio de curso les ponía problemas de su nivel, y lo que me decían era: se suma, se resta. Entonces poco a poco, les he ido inculcando que cuando me resuelvan un problema me hagan una ficha. Lee el problema, y ahora piensa la primera pregunta que te tienes que hacer. Además, les exijo, casi siempre, que me la escriban. Porque ése es otro fallo, el niño si lo dice se le va, cuando se da cuenta es cuando lo está escribiendo. Casi siempre les he exigido que me lo escriban. ¿Que primera pregunta hace el problema? ¿Lo sabes contestar? Lee el problema y averígualo. Pide que cuántos $\mathrm{Km}$ he recorrido. Y me lo escribe. La segunda pregunta es: ¿qué datos conozco del problema?, ¿qué cosas me dice?, me ponen los datos, y luego para llegar a esa pregunta que te ha hecho, el camino. ¿Qué necesito saber? Me van poniendo: $1^{9}$ ) necesito saber los litros de gasolina que gasto, $2^{\circ}$ ) nece- 
sito saber lo que sea. Y así he conseguido que la mayoría de ellos resuelvan un problema; tienen fallos por supuesto, y hay algunos, concretamente 2 o 3 que todavía no he conseguido que me resuelvan el problema.»

El conocimiento que esta profesora tiene sobre el proceso de resolución de problemas (Blanco 1991b) ha sido generado, al igual que su visión sobre la enseñanza de las Matemáticas, a partir de su propia experiencia, y así nos Io manifiesta expresamente en una entrevista que trataba específicamente sobre la resolución de problemas:

«Desde un punto de vista elemental, me llamó la atención ver que el niño no sabía hacerse la pregunta. A partir de esa fase en la que, más o menos, ellos saben qué es lo que querían averiguar con el problema, hubo otra fase que también fue difícil para ellos: darse cuenta de los datos que les daban para averiguar aquello y saber cuándo está terminado el problema. Eso lo tuve que ir haciendo poco a poco, durante el curso pasado. Incluso ahora, hay un grupito al que todavía les cuesta mucho resolver problemitas sencillos. No saben, o no tienen suficiente atención porque nada más que hacen y hacen. Todavía no están maduros. Consideré las tres fases complementarias, averiguar qué te pide el problema, saber los datos y saber los caminos que tienes que seguir hasta llegar al final. $Y$ ver que averiguado lo que me piden, ya está resuelto».

Por último quisieramos recordar la manifestación expresa que nuestra informante realiza sobre su propio aprendizaje, que pone de manifiesto el proceso de autoformación seguido.

«Con los problemas, yo he tenido un problema. Hasta que he llegado a esta situación, han pasado años pensando cómo enseñaría a los muchachos a resolver problemas. ¿Qué esquema les daría, qué orden seguir? Hasta que he llegado a esta solución. Que coincide con lo que dícen por ahí los matemáticos, pero que yo no lo hábía oído».

\section{DISCUSIÓN}

A través de estos dos ejemplos concretos hemos querido evidenciar que existen conexiones entre el conocimiento práctico personal de nuestros profesores expertos y las aportaciones que están siendo consideradas para la formación de los alumnos en las escuelas de Magisterio, tomadas de textos clásicos de Didáctica de las Materráticas, y cuyos postulados tienen su origen en la práctica educativa.
De igual manera, podríamos haber señalado otros momentos de la enseñanza desarrollada por nuestros expertos que tienen relación con otros contenidos que desarroIlamos en nuestras clases (Blanco 1991a, p. 255). A este respecto, y a modo de ejemplo, hemos encontrado evidencias sobre el significado de las Matemáticas como medio de comunicación, en relación con el significado que aparece en Cockroft (1985) o en Dorfler y Mclone (1986), o sobre los factores que hay que considerar en la resolución de problemas (Charles y Lester 1982).

El estudio de estas relaciones aportará elementos descriptivos y metodológicos que facilitará la comprensión del contexto docente y, consecuentemente, ayudará a establecer líneas generales para contextualizar la enseñanza de las Matemáticas en el nivel señalado.

La búsqueda de ejemplos concretos que surjan de la práctica docente sobre los que fundamentar nuestras disertaciones, así como el establecimiento de conexiones entre los contenidos impartidos en la asignatura de Didáctica de las Matemáticas y la práctica desarrollada por los profesores con experiencia, puede ser un camino interesante que nos ayude a mejorar los contenidos curriculares en la formación inicial de los profesores que imparten Matemáticas en la EGB.

La relación entre teoría y práctica de la Didáctica de las Matemáticas, a través de los estudios de casos, puede llevarnos, a los responsables de la formación inicial, a establecer pautas de reflexión y acción que ayuden a nuestros alumnos a la comprensión de la actividad docente impartida por nuestros profesores con experiencia. Al mismo tiempo, y dadas las dificultades encontradas para que nuestros alumnos aprendan de su propia interacción didáctica, les proporcionaríamos experiencias a partir de las cuales podrian construir una estructura de conocimiento que les ayudara a identificar la información que les fuera útil durante la realización de sus prácticas docentes (Livingston y Borko 1989).

Por otra parte, los métodos cualitativos de investigación utilizados en el trabajo son un elemento para la reflexión de los profesores que han participado en la investigación. El trabajo realizado ha ayudado a nuestros informantes a reflexionar sobre su propia experiencia educativa, mostrándose ellos mismos satisfechos de su participación, lo que ya de principio aporta una justificación para la labor reailizada, y muestra que la metodología cualitativa es válida para los programas de Formación permanente del profesorado. Como consecuencia, esta metodología se nos manifiesta especialmente importante para ayudar a los profesores a reflexionar y profundizar sobre su pensamiento y acción, poniendo de manifiesto su conocimiento práctico personal. 


\section{REFERENCIAS BIBLIOGRÁFICAS}

BLANCO, I.., 1991 a. Conocimiento y acción en la enseñanza de las Matemáticas, de profesores de EGB y estudiantes para profesores. (Servicio de Publicaciones de la Universidad de Extremadura: Badajoz).

BLANCO, L., 1991b. La resolución de problemas y los profesores de Matemáticas, Suma, 9, pp. 32-39.

CALDERHEAD, J., 1981. Stimulated recall. A method for research on teaching, British Journal of Educational Psychology, 51, pp. 180-190.

CHARLES, R. y LESTER, F., 1982. Teaching problem solving. What, Why, How. (Dale Seymour Pu.: Palo Alto).

COCKROFT, Informe, 1985. Las Matemáticas sí cuentan. (MFC: Madrid).

DIENES, Z.P., 1970. La construcción de las Matemáticas. (Vicens-Vives: Barcelona).

DORFI,ER, W, y McLONE, R. R., 1986. Mathematics as a school subject, in Christiansen, Howson y Otte, Perspectives on Mathematics education, pp. 49-97. (Reidel Pub. Co.: Dordrecht, Holland).

GAULIN, C., 1986. Tendencias actuales en la enseñanza de las matemáticas l. Números, 14. pp. 11-18.

LIVINGSTON, C. y BORKO, H., 1989. Expert-Novice differences in teaching: a cognitive analysis and impli- cations for teacher education, Journal of Teacher Education, Vol, XXXX (4), Pp. 36-43.

MARCELO, C., 1987. El pensamiento del profesor, (Ceac: Barcelona).

MARKS, R., 1989. What exactly is pedagogical content knowledge? Examples from Mathematics, (AERA: San Francisco).

POLYA, G., 1949. On solving Mathematical problems in High School, California Mathematics Council Bulletin, 7(2).

POLYA, G., 1985. Cómoplanteary resolver problemas. (Trillas: México) $13^{?}$ ed.

SCHROEDER, T.L. y LESTER. F.L., 1989. Developing understanding in mathematics via problem solving, in NCTM, New directions for elementary school mathematics, pp. 31-42. (NCTM: Virginia).

SHAVELSON, R.J. y STERN, P., 1983. Investigación sobre el pensamiento pedagógico del profesor, sus juicios, decisiones y conductas, en Gimeno, J. y Pérez Gómez, A. La enseñanza, su teoría y su práctica, pp. 372-419, (Akal: Madrid).

WOODS, P., 1987. La escuela por dentro, (Paidós-MEC: Barcelona). 